\title{
Real-Time 3D Super-Resolution Fluorescence Lifetime Imaging Microscopy, in vivo
}

\section{Scott Howard}

\author{
University of Notre Dame, Notre Dame, IN, Indiana, United States
}

Fluorescence lifetime imaging microscopy (FLIM) has enabled additional molecular imaging contrast as well as quantitative measurements (e.g., FRET, collisional quenching, protein binding, and pH). When combined with intravital imaging techniques, such as multiphoton microscopy (MPM), three dimensional FLIM is possible. While intravital FLIM provides a wealth of information, most techniques are relatively slow (can take seconds to minutes to acquire an image), can require high excitation dosages (potentially prohibitively high for long term in vivo imaging), and are limited by the same spatial resolution limits present in microscopy. This presentation will describe both the fundamental and technical limitations to imaging speed and use that knowledge to design and experimentally demonstrate performance of a new "Instant FLIM." The Instant FLIM platform is capable of real-time imaging and phasor analysis of a sample at the same image acquisition speed as an MPM microscope. The system is a true "real-time" FLIM microscope as the lifetime and phasor computation time is performed faster than the image acquisition time using a custom designed analog computer, thereby reducing digital sampling bandwidth requirements and eliminating the need for curve fitting to extract fluorescence lifetime. The system is analogous to software defined radio where a phase modulated signal is acquired and measured in quadrature (i.e., measuring the sine and cosine components of the signal at the fundamental frequency), from which the underlying phase changes can be directly measured with little computation. Since frequency domain FLIM is essentially a phase modulated signal, this approach enables high speed analysis in the phasor domain, natively. By leveraging these commonly used techniques from radio frequency (RF) engineering, the "Instant FLIM" system is implemented as an add-on module to an MPM microscope using mature and widely available RF components for $\sim \$ 2500$.

This high speed FLIM system is demonstrated in in vivo mouse and zebrafish brains and spinal cords. The system is also compatible with generalized stepwise optical saturation (GSOS), a technique to perform superresolution microscopy in FD-FLIM. The technique is the point-scanning analog of nonlinear structured illumination, and has been extended to modulation phase-sensitive super-resolution MPM-FLIM.

\section{References}

Zhang, Y., Guldner, I., Nichols, E., Benirschke, D., Smith, C., Zhang, S., and Howard, S. "Instant FLIM Enables 4D in Vivo Lifetime Imaging of Intact Brains" bioRxiv (2020)

Zhang, Y., Benirschke, D., Abdalsalam, O., and Howard, S. S. "Generalized Stepwise Optical Saturation Enables Super-Resolution Fluorescence Lifetime Imaging Microscopy” Biomed. Opt. Express 9, no. 9 (2018) 